\title{
The drug discovery factory: An inevitable evolutionary consequence of high-throughput parallel processing
}

\author{
Richard Archer
}

Recent advances in drug discovery technology have led to significant progress in accelerating drug target identification, lead generation, lead optimization, and the development of clinical drug candidates. Although significant, this is not spectacular progress and many wonder why. What will it take for pharmaceutical companies to realize a dramatic expansion of their product pipelines, and to bring to the marketplace novel drugs that can dominate the market and command a premium price in the highly competitive environment of managed care and tightening cost controls? What is needed for drug companies to be able to justify huge expenditures on advanced technology and highthroughput processing systems?

For drug discovery to meet the demands of the 21st century, to produce safe, high quality, and targeted new drugs that address unmet market needs and are tailored to the growing knowledge base of human genetic variation, a restructuring of the drug discovery process will be necessary. This overhaul of the system will take the current researchoriented and innovation-driven process and apply to it, in both theory and practice, the principles of industrial-scale manufacturing. It will represent a shift from the uncertainty of scientific inquiry and the pioneering mentality embodied in a research campus, to the finely tuned, logistics-based organizational culture of a production-scale operation.

The focal point of this new, industrialized era in pharmaceutical discovery will be the drug discovery factory, a stand-alone facility that effectively implements state-of-the-art automation in a computer-controlled system. This facility might stand just across the street from a pharmaceutical company's research laboratories, and its work will flow fluidly from that core of scientific discovery; however, to operate effectively, it needs to remain physically and philosophically distinct, maintaining an organizational structure and management style that befits an industrial manufacturing plant. This industrialization of drug discovery, the inevitable "next step" in the evolution of today's compound synthesis and screening effort, will advance high-throughput parallel processing

Richard Archer is chief executive of The Automation Partnership, Melbourn Science Park, Melbourn, Royston SG8 6HB, UK. Email:jra@autoprt.co.uk. to new levels and will enable the drug discovery engine to achieve its full potential.

During the past two decades, each component of the drug discovery machine has made steady advances, punctuated by bursts of technological innovation. These bursts have given us, for example, the genomics revolution and an abundance of potential drug targets, combinatorial chemistry and seemingly boundless compound libraries, as well as high-throughput screening and the rapid parallel processing of infinite target and compound combinations to generate "hits"high quality hits being the measure of success of the overall drug discovery process. Facilitating all of these achievements has been the explosive growth in the fields of computer hardware and software (the latter spawning the discipline of bioinformatics), automation technology, and miniaturization strategies.

But despite the relatively recent success of several bioengineered drugs, and the growing

\section{High-throughput screening}

has not yet demonstrated

\section{that it can produce the} number of hits of which it is

\section{theoretically capable.}

drug pipelines of the major pharmaceutical companies, the drug discovery machine is still far from achieving its potential, far from churning out a steady stream of high quality hits. As each component has reached new heights in speed and productivity, the bottleneck in the system has simply shifted to a different part of the process.

High-throughput screening in particular, in which the industry has invested millions of dollars, has not yet demonstrated that it can produce the number of hits of which it is theoretically capable. The ultrahigh-throughput systems currently in development promise increased data output; however, when viewed in isolation, the upgrading of a single component of the process, such as high-throughput screening, may not yield a significant impact on the productivity of the system as a whole.

Consider, for example, if you traded in your family's minivan for a Formula One racing car. Would your daily trip to the office take any less time? Not really, if you abide by the speed restrictions, traffic rules, and other limitations imposed by the infrastructure of the existing highway system. To truly take advan- tage of your new-found speed requires a carefully planned increase in the complexity and sophistication of the entire system.

The drug discovery engine, to shift into high gear, needs to scale-up its entire operation. And not simply in a linear fashiondoing more of the same, building higher-volume and faster systems, or miniaturizing processes and their components-but by incorporating, in principle and in practice, the fundamentals of automation, process engineering, and logistics-based management. High-throughput screening, to be truly effective, should be based on more than the application of robotics technology to drug screening. As a critical component of a drug discovery factory, high-throughput screening should be able to perform 100,000 assays/day, not just for one day, but every day. For this to occur, though, the entire system must be able to operate at maximum capacity: 100,000 assays requires 100,000 compounds collected and prepared in the proper format and at the prescribed concentrations, multiple targets in varying assay formats at the ready, and all assay reagents selected and available.

The robots, analyzers, and other instrumentation must be capable of operating 24 hours/day for weeks at a time, with minimal, scheduled off-line time for maintenance and repairs. Flexibility and responsiveness must be built in to the initial design and construction of the process, with high-throughput screening systems being readily adaptable to perform in vitro as well as functional, cellbased assays using a variety of targets, and to quickly accommodate novel assay formats and new technologies.

Computerized control networks linked to complex data management systems could oversee the entire facility. An industrialized process calls for unmanned operations, with computers running the factory, leaving scientists free to pursue scientific endeavors.

Drug discovery can learn from the experiences of other industries that have, over the years, optimized their manufacturing operations. The automotive industry and silicon chip producers are excellent examples of a versatile approach to manufacturing that relies on modular robotic systems, high-speed parallel processing, and a highly integrated control system. Adopting this approach, and adapting it to meet the specific demands of drug development, will result in a drug discovery factory that can produce novel drug candidates on a truly grand scale. 